\title{
Blastocystis Hominis Infection Pathogenic or Commensal: Short Review
}

\author{
Sonu Kumari Agrawal \\ Department of Microbiology, AIIMS, New Delhi, India
}

*Corresponding Author: Sonu Kumari Agrawal

\begin{abstract}
Blastocystis is anaerobic, unicellular protozoan resides in intestinal tract of human as well as other hosts. Despite the various observations over the year, the classification of Blastocystis Species still controversial and it is difficult to define its taxonomical status. There are variations in morphological forms of Blastocystis hominis but three major forms commonly noted: vacuolar, granular, ameboid. The pathogenicity of Blastocystis is still matter of debate. A number of studies have shown the pathogenic potential of this organism. Hence, in this review, we will discuss classification, morphology, lifecycle, pathogenicity, Clinical features, Diagnosis and treatment of Blastocystis.
\end{abstract}

Keywords: Blastocystis hominis, protozoa, commensal, pathogen

\section{INTRODUCTION}

Blastocystis is anaerobic, unicellular protozoan resides in intestinal tract of human as well as other hosts. It was considered as fungus before, now it has been classified to a protozoa. The pathogenicity of the organism is still doubtful and is the subject of debate. Hence, in this review, we will discuss classification, morphology, lifecycle, pathogenicity, Clinical features, Diagnosis and treatment of Blastocystis.

\section{Classification}

Despite the various observations over the year, the classification of Blastocystis Species still controversial and it is difficult to define its taxonomical status. It was Alexeieff and Brumpt, who proposed that Blastocystis is saprophytic yeast of the intestinal tract. ${ }^{1}$ The genus name "Blastocystis" was proposed by Alexeieff and term "hominis" was proposed by Brumpt because it was isolated from human faeces. ${ }^{2}$ Later, Zierdt et.al gave an evidence that organism was not fungus and on the basis of morphological properties : presence of one or more nuclei, Golgi apparatus, endoplasmic reticulum, mitochondria like organelles and physiological properties such as failure to grow on fungal medium resistant to antifungal and antibacterial and sensitive to antiprotozoal drugs, he classified the organism as a Protists. ${ }^{3}$ Small-subunit rRNA molecular sequencing techniques provided an evidence that Blastocystis hominis is not related to fungi and sporozoans. ${ }^{4}$ Later on Jiang et.al, reclassified the organism into the class Blastocystea, the order Blastocystida,the family Blastocystidae, the genus Blastocystis and type species hominis. ${ }^{5}$ Designation of the organism to species level is still not adequately resolved. ${ }^{6}$ Further studies are required for morphological and molecular data to resolved the issues.

\section{MORPHOLOGY}

There are variations in morphological forms of Blastocystis hominis but three major forms commonly noted: vacuolar, granular, ameboid. ${ }^{1,3,7}$ In addition other forms such as multivacuolar, avacuolar and cysts forms have also been described. ${ }^{8}$

\section{Vacuolar form:}

It is most predominant and typical form used for diagnosis of Blastocystis hominis. In this morphological form size ranging from 2 to $200 \mu \mathrm{m}$ in diameter. ${ }^{9}$ This form consist of large central vacuole with thin peripheral rim. This peripheral rim contains one or more than one nuclei. ${ }^{10}$ On wet 
mount examination Blastocystis hominis appear as central empty space hence it is known as vacuolar form. ${ }^{11}$ Later on it was found that these vacuoles are membrane bound bodies hence it is also known as central body containing granular material. ${ }^{12-14}$ These central body act as storage organelle and plays a role in apoptosis of organism. ${ }^{15}$ Vacoular form undergo encystations to form cysts. ${ }^{16}$

\section{Granular form:}

This form is morphologically similar to vacuolar form except that central vacuole contains granules. Granular forms are larger than vacuolar form with size vary from 10 to $60 \mu \mathrm{m}$ in diameter. ${ }^{3}$ There are three types of granules have been described in literature - lipid, metabolic and reproductive granules. The lipid granules are present in cytoplasm and act as storage granules. ${ }^{17}$ The metabolic granules are involved in metabolic pathways and reproductive granules play role in schizogony. ${ }^{18,19}$

\section{Ameboid form}

The ameboid form is rarely reported.$^{17}$ The central vacuole is absent in this form. They are $10 \mu \mathrm{m}$ in diameter, irregular in shape. Because of their small they often get confused with neutrophils and macrophages. ${ }^{19}$ They have pseudopods but are non-motile. ${ }^{17}$

\section{Cyst form}

The cyst forms are smaller in diameter ( 3 to $6 \mu \mathrm{m}$ ) as compare to vacuolar and granular form. ${ }^{20}$ They are spherical and surrounded by multilayered thick wall. The cyst contain nuclei vary from 1 to $4 .^{21}$ The cyst are thought to protect the parasite under adverse conditions.

\section{Mode of Transmission and Life Cycle}

It is mainly transmitted thorough faeco-oral route. ${ }^{22}$ Some of the studies have shown that ingestion drinking water, raw vegetables or fruits and unclean hands contaminated with cysts can serve as transmission of Blastocystis infections. ${ }^{23,24}$ After ingestion of cysts, it undergoes excystation to differentiate into vacuolar form in large intestine. ${ }^{25}$ Then, vacuolar forms can differentiate into other forms such as multi-vacuolar, avacuolar and amoeboid ${ }^{26}$ In the lumen of intestine vacuolar forms encyst to cysts which are passed in the stools .Binary fission is most accepted mode of reproduction in vacuolar forms in this organisms. ${ }^{27}$

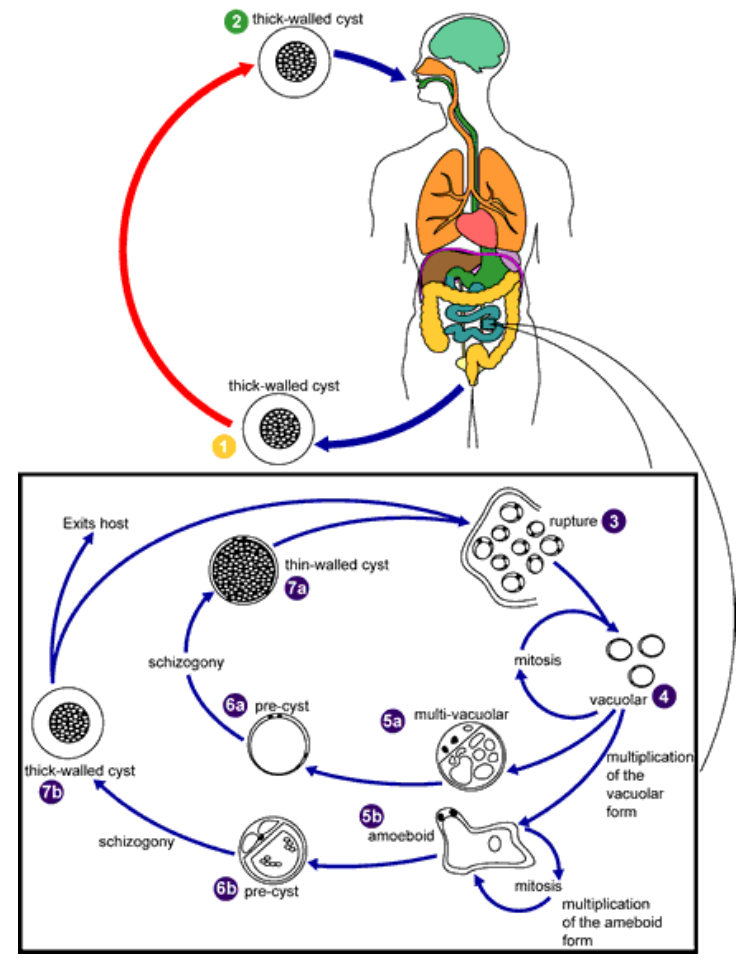

\section{Pathogenicity}

The pathogenicity of Blastocystis is still matter of debate. A number of studies have shown the pathogenic potential of this organism. ${ }^{28}$ Due to absence of proper animal model exact pathogenic mechanism is not known. ${ }^{28}$ The most convincing result to explain pathogenicity is relation of virulence 
with subtype pathogenicity (ST). ${ }^{29}$ Subtype $1,2,3,4,6$ have been reported from symptomatic patients. ${ }^{30,31}$ The most common subtype is ST $3 .^{31,32}$ The most studied morphological form is amoeboid in pathogenic members. ${ }^{33,34}$ Subtype alone does not explain the pathogenicity. Hydrolytic enzymes and proteases secreted by Blastocystis lead to secretion of interleukin- 8 by activating the gut mucosa, responsible for gastrointestinal symptoms. ${ }^{34}$ However, the factors responsible for extraintestinal symptoms are not yet known. More genomic studies are required to confirm the pathogenicity of Blastocystis.

\section{Clinical Features}

The person can present with no symptoms or with intestinal and extra-intestinal symptoms.

\begin{tabular}{|l|l|l|}
\hline Asymptomatic & Intestinal & Extra-intestinal \\
\hline $\begin{array}{l}\text { Some of studies have found } \\
\text { Vacuolar forms in stool of } \\
\text { asymptomatic patients. }\end{array}$ & $\begin{array}{l}\text { Abdominal pain, diarrhea, nausea, } \\
\text { rectal bleeding, hepatomegaly, } \\
\text { spleenomegaly. }\end{array}$ & Joint pain, swelling, arthritis. \\
\hline
\end{tabular}

\section{Laboratory Diagnosis}

Blastocystis shows polymorphism and vacuolar, granular, ameboid, cyst, all forms can be identified in faecal sample .Concentration techniques are not useful in case of Blastocystis infection due to disruption of morphology of organism. ${ }^{35}$ Multiple stool samples should be examined to enhance the detection rate of parasites. ${ }^{36}$ Microscopy is most widely used method of examination. In wet mount unstained or stained with iodine examination organisms are round approximately 2 to $>200 \mu \mathrm{m}$ in diameter having large central vacuole surrounded by one or multiple nuclei. ${ }^{4}$ Other staining methods such as Trichrome, Giemsa, Gram and Wright's have been found to succeed. ${ }^{1,37,38}$ Electron microscopy is not generally required for routine diagnosis but useful to confirm the atypical morphological forms of parasites. Culture methods are more sensitive than direct smear examination .Culture media such as Drbohlav egg medium, modified Dulbecco's medium $+10 \%$ horse serum, minimal essential medium (MEM) $+10 \%$ horse serum, Diamond's trypticase serum monophasic media have shown successful cultivation of organisms anaerobically at $37^{0} \mathrm{c} .{ }^{39}$ Blastocystis infections can be detected by serological techniques such as ELISA (Enzyme linked immunosorbent assay and IFA (Immunofluoroscent assay) but these immunological techniques are not use in routine diagnosis. ${ }^{40}$ Studies have found that successful use of Polymerase chain reaction (PCR) for diagnosis of Blastocystis infections.

\section{Treatment}

The need to treat Blastocystis infections is still controversial. It is thought that infection is self limited and intervention may not be warranted. However, some believe that treatment is required in debilitating cases. ${ }^{4}$ Antiprotozoal drugs mainly metronidazole or iodoquinol are recommended for management infections. ${ }^{38,41}$ Dose of metronidazole is 250 to $750 \mathrm{mg}$ three times per day for 5 to 10 days and iodoquinol is $300 \mathrm{mg}$ three times per day for 10 days is recommended for treatment of infections. ${ }^{42-44}$

\section{CONCLUSiON}

The role of Blastocystis (unicellular protozoan parasite) is still matter of debate. Due to lack of suitable animal model exact virulence factors are not known so, more studies are required to explain the pathogenic role of Blastocystis in human diseases.

\section{REFERENCES}

[1] Zierdt CH. Blastocystis hominis: Past and future. Clin Microbiol Rev. 1991;4:61-79

[2] Brumpt, E. Co`lite a`Tetramitus mesnili (Wenyon 1910) et co`lite a` Trichomonas intestinalis Leuchart 1879. Blastocystis hominis n. sp. et forms voisines. Bull. Soc. Pathol. Exot.1992 5:725730

[3] Zierdt CH, Rude WS, Bull BS. Protozoan characteristics of Blastocystis hominis. Am J Clin Pathol.1967; 48:495-501.

[4] Stenzel DJ, Boreham PF, Blastocystis hominis Revisited Clin Microbiol Rev. 1996 Oct;9(4):56384

[5] Jiang, J.-B., and J.-G. He. Taxonomic status of Blastocystis hominis. Parasitol. Today 1993 9:23. 
[6] Noël C, Dufernez F, Gerbod D, Edgcomb VP, Delgado-Viscogliosi P, Ho LC, et al. Molecular phylogenies of Blastocystis isolates from different hosts: Implications for genetic diversity, identification of species, and zoonosis. J Clin Microbiol. 2005;43:348-55

[7] Boreham, P. F. L., and D. J. Stenzel. Blastocystis in humans and animals: morphology, biology, and epizootiology. Adv. Parasitol. 1993.32:1-70

[8] Stenzel DJ, Boreham PF, McDougall R. Ultrastructure of Blastocystis hominis in human stool samples.Int J Parasitol. 1991;21:807-12

[9] Zierdt, C. H., and H. Tan.Endosymbiosis in Blastocystis hominis. Parasitol.1976: 39:422-430

[10] MacPherson DW, MacQueen WM. Morphological diversity of Blastocystis hominis in sodium acetate-acetic acid-formalin-preserved stool samples stained with iron hematoxylin. J Clin Microbiol. 1994;32:267-8

[11] Parija SC, Jeremiah S.Trop Parasitol. Blastocystis: Taxonomy, biology and virulence. 2013 Jan;3(1):17-25

[12] Dunn LA, Boreham PF, Stenzel DJ. Ultrastructural variation of Blastocystis hominis stocks in culture. Int J Parasitol. 1989;19:43-56

[13] Yoshikawa H, Kuwayama N, Enose Y. Histochemical detection of carbohydrates of Blastocystis hominis. J Eukaryot Microbiol. 1995;42:70-4

[14] Yoshikawa H, Satoh J, Enose Y. Light and electron microscopic localization of lipids in Blastocystis hominis. J Electron Microsc (Tokyo) 1995;44:100-3

[15] Nasirudeen AM, Tan KS, Singh M, Yap EH. Programmed cell death in a human intestinal parasite,Blastocystis hominis. Parasitology. 2001;123:235-46

[16] Zhang X, Qiao JY, Dong XH, Li YQ, Li XQ, Li C. Study on morphology of Blastocystis hominis in culture and from diarrhea patients. Zhongguo Ji Sheng Chong Xue Yu Ji Sheng Chong Bing Za Zhi.2003;21:116-8

[17] Tan HK, Zierdt CH. Ultrastructure of Blastocystis hominis. Z Parasitenkd. 1973;42:315-24

[18] Suresh K, Howe J, Ng GC, Ho LC, Ramachandran NP, Loh AK, et al. A multiple fission-like mode of asexual reproduction in Blastocystis hominis. Parasitol Res. 1994;80:523-7

[19] Zierdt, C. H. Blastocystis hominis, a long-misunderstood intestinal parasite. Parasitol. Today 1988: 4:15-17.

[20] Zaman, V., J. Howe, and M. Ng. Ultrastructure of Blastocystis hominis cysts. Parasitol. Res.1995 81:465-469.

[21] Stenzel, D. J., and P. F. L. Boreham. A cyst-like stage of Blastocystis hominis. Int. J. Parasitol.1991.21:613-615

[22] Yoshikawa H, Yoshida K, Nakajima A, Yamanari K, Iwatani S, Kimata I. Fecal-oral transmission of the cyst form of Blastocystis hominis in rats. Parasitol Res. 2004;94:391-6

[23] Anuar TS, Ghani MK, Azreen SN, Salleh FM, Moktar N. Blastocystis infection in Malaysia: Evidence of waterborne and human-to-human transmissions among the Proto-Malay, Negrito and Senoi tribes of Orang Asli. Parasit Vectors. 2013;6:40

[24] Li LH, Zhou XN, Du ZW, Wang XZ, Wang LB, Jiang JY, et al. Molecular epidemiology of human Blastocystis in a village in Yunnan province, China. Parasitol Int. 2007;56:281-6

[25] Moe KT, Singh M, Howe J, Ho LC, Tan SW, Chen XQ, et al. Experimental Blastocystis hominis infection in laboratory mice. Parasitol Res. 1997;83:319-25

[26] Zhang X, Zhang S, Qiao J, Wu X, Zhao L, Liu Y, et al. Ultrastructural insights into morphology and reproductive mode of Blastocystis hominis. Parasitol Res. 2012;110:1165-72

[27] Moe KT, Singh M, Howe J, Ho LC, Tan SW, Chen XQ, et al. Development of Blastocystis hominis cysts into vacuolar forms in vitro. Parasitol Res. 1999;85:103-8

[28] Scanlan PD. Blastocystis: Past pitfalls and future perspectives. Trends Parasitol. 2012;28:327-34

[29] Tan KS, Singh M, Yap EH. Recent advances in Blastocystis hominis research: Hot spots in terra incognita. Int J Parasitol. 2002;32:789-804

[30] Tan KS, Singh M, Yap EH. Recent advances in Blastocystis hominis research: Hot spots in terra incognita. Int J Parasitol. 2002;32:789-804 
[31] Dogruman-Al F, Kustimur S, Yoshikawa H, Tuncer C, Simsek Z, Tanyuksel M, et al. Blastocystis subtypes in irritable bowel syndrome and inflammatory bowel disease in Ankara, Turkey. Mem Inst Oswaldo Cruz. 2009;104:724-7

[32] Moosavi A, Haghighi A, Mojarad EN, Zayeri F, Alebouyeh M, Khazan H, et al. Genetic variability of Blastocystis sp. isolated from symptomatic and asymptomatic individuals in Iran. Parasitol Res.2012;111:2311-5

[33] Tan TC, Suresh KG, editors. Predominance of amoeboid forms of Blastocystis hominis in isolates from symptomatic patients. Parasitol Res. 2006;98:189-93

[34] Abdel-Hameed DM, Hassanin OM. Proteaese activity of Blastocystis hominis subtype 3 in symptomatic and asymptomatic patients. Parasitol Res. 2011;109:321-7

[35] Miller, R. A., and B. H. Minshew. Blastocystis hominis: an organism in search of a disease. Rev. Infect. Dis. 1988.10:930-938

[36] Guimara es, S., and M. I. L. Sogayar. Blastocystis hominis: occurrence in children and staff members of municipal day-care centres from Botucatu, Sa o Paulo State, Brazil. Mem. Inst. Oswaldo Cruz Rio J. 1993.88:427-429

[37] Ricci, N., P. Toma, M. Furlani, M. Caselli, and S. Gullini. 1984. Blastocystis hominis: a neglected cause of diarrhoea? Lancet i:966.

[38] Vannatta, J. B., D. Adamson, and K. Mullican. Blastocystis hominis infection presenting as recurrent diarrhea. Ann. Intern. Med.1985. 102:495-496

[39] Al FD, Hökelek M Is Blastocystis hominis an opportunist agent? Turkiye Parazitol Derg 2007;31(1):28-36.

[40] El Masry, N. A., S. Bassily, Z. Farid, and A. G. Aziz. 1990. Potential clinical significance of Blastocystis hominis in Egypt. Trans. R. Soc. Trop. Med. Hyg. 84:695

[41] Waghorn, D. J., and P. Hancock.Clinical significance of Blastocystis hominis. Lancet 1991.337:609.

[42] Guirges, S. Y., and N. S. Al-Waili. Blastocystis hominis: evidence for human pathogenicity and effectiveness of metronidazole therapy. Clin. Exp. Pharmacol. Physiol. 1987.14:333-335

[43] Hussain Qadri, S. M., G. A. Al-Okaili, and F. Al-Dayel. Clinical significance of Blastocystis hominis. J. Clin. Microbiol. 1989.27:2407-2409.

[44] Zafar, M. N. Morphology and frequency distribution of protozoan Blastocystis hominis. J. Pakistan Med. Assoc. 1988.38:322-324

Citation: S. K. Agrawal, "Blastocystis Hominis Infection Pathogenic or Commensal: Short Review ", International Journal of Research studies in Microbiology and Biotechnology, vol. 3, no. 3, p. 5, 2017. http://dx.doi.org/10.20431/2454-9428.0303001

Copyright: (C) 2017 Authors. This is an open-access article distributed under the terms of the Creative Commons Attribution License, which permits unrestricted use, distribution, and reproduction in any medium, provided the original author and source are credited. 